\title{
Existence of Solitary Waves in a Perturbed KdV-mKdV Equation
}

\author{
Chengqun Li $\mathbb{D},{ }^{1}$ Minzhi Wei $\mathbb{D},{ }^{1}$ and Yuanhua Lin $\mathbb{D}^{2}$ \\ ${ }^{1}$ Deparment of Applied Mathematics, Guangxi University of Finance and Economics, Nanning, Guangxi 530003, China \\ ${ }^{2}$ School of Mathematics and Statistics, Hechi University, Yizhou, Hechi, Guangxi 546300, China
}

Correspondence should be addressed to Yuanhua Lin; yuanhualin01@163.com

Received 6 April 2021; Revised 3 May 2021; Accepted 19 May 2021; Published 28 May 2021

Academic Editor: Efthymios G. Tsionas

Copyright $\odot 2021$ Chengqun Li et al. This is an open access article distributed under the Creative Commons Attribution License, which permits unrestricted use, distribution, and reproduction in any medium, provided the original work is properly cited.

In this paper, we establish the existence of a solitary wave in a $\mathrm{KdV}-\mathrm{mKdV}$ equation with dissipative perturbation by applying the geometric singular perturbation technique and Melnikov function. The distance of the stable manifold and unstable manifold is computed to show the existence of the homoclinic loop for the related ordinary differential equation systems on the slow manifold, which implies the existence of a solitary wave for the KdV-mKdV equation with dissipative perturbation.

\section{Introduction}

$\mathrm{KdV}$-mKdV equation plays a most important role in soliton physics and appears in many practical scenarios such as thermal pulse and wave propagation of the bound particle [1]. Recently, many mathematicians are interested in studying traveling wave solutions for the $\mathrm{KdV}-\mathrm{mKdV}$ equation, and there are many tools to find the traveling wave solutions, such as the inverse scattering method [2], the double subequation method [3], the sine-cosine method [4, 5], the Darboux transformation [6], the Exp-expansion method [7, 8], and the bifurcation method of dynamic systems [9]. KdV-mKdV equation is one of the most popular soliton equations; therefore, there were widely related investigations in [10-14]. On the contrary, based on the relationship between the solitary wave solution and the homoclinic orbit of the associated ordinary differential equations, Fan and Tian [15] proved that the solitary wave persists the singularly perturbed $\mathrm{mKdV}-\mathrm{KS}$ equation from the geometric singular perturbation point of view, when the perturbation parameter is suitably small. Mansour proved the existence of traveling wave solutions of some singular perturbed equations [16] by using the geometric singular perturbation theory. Yan et al. [17] investigated a perturbed generalized $\mathrm{KdV}$ equation. They proved the existence of solitary waves and periodic waves by applying the geometric singular perturbation theory and the regular perturbation analysis for a Hamiltonian system.
Solitary waves are a special form of ultrashort pulses, which maintain a constant shape, amplitude, and velocity during their propagation. From the point of view of physics, solitary wave is a special product of the nonlinear effects of matter. Mathematically, it is a class of stable, energy-finite, nondispersive solutions of some nonlinear partial differential equations. As the particular characteristics of the solitary wave, we shall use Fenichel persistence theorem $[18,19]$ to study the existence of the solution for the singularly perturbed $\mathrm{KdV}-\mathrm{mKdV}$ equation in this paper.

$$
U_{t}+U^{p} U_{x}+U^{q} U_{x}+U_{x x x}+\varepsilon\left(U_{x x}+U_{x x x x}\right)=0,
$$

where $p, q \in Z^{+}$and $p<q$, not studied by the same method before. We try to investigate the existence problem of solitary wave solutions of (1) by using the geometric singular perturbation technique.

\section{Fenichel Theory}

In this section, we review the necessary theory that we will use for our discussion. We take the exposition in Christopher [19]. For details, one can resort to Fenichel [18] or Jones [19]. Geometric singular perturbation theory was first given by Fenichel and is often referred to as Fenichel theory. A comprehensive overview of the theory, as well as new proofs of many theorems and detailed examples of applications, was given by Jones [19]. 
Consider the standard fast-slow system:

$$
\left\{\begin{array}{l}
\dot{f}(t)=F(f(t), s(t), \varepsilon), \\
\dot{g}(t)=\varepsilon G(f(t), g(t), \varepsilon),
\end{array}\right.
$$

where is the derivative to $t, 0<\varepsilon \ll 1$ is a real parameter, $f=\left(f_{1}, f_{2}, \ldots, f_{n_{f}}\right)^{T} \in R^{n_{f}}, \quad g=\left(g_{1}, g_{2}, \ldots, g_{n_{g}}\right)^{T} \in R^{n_{g}}$, $n_{f}+n_{g}=n, \max \|\dot{f}\|=\max \|\dot{g}\|$, and $f$ corresponds to fast directions and $g$ corresponds to slow directions. $f, g$ are $C^{\infty}$ on the set $U \times V$, where $U \subset R^{n}$ and $V$ is an open interval containing 0 . Assume that, for $\varepsilon=0$, the system has a compact normal hyperbolic manifold $M_{0}$ which contained in the set $f(x, y, 0)=0$. The manifold $M_{0}$ is hyperbolic normally if the linearization of (2) at each point in $M_{0}$ has exactly $n_{g}$ eigenvalues with zero real part, where $n_{g}$ is the dimension of the center point.

Fenichel's Persistence Theorem 1. Under the assumption described above, if $\varepsilon>0$ is small sufficiently, there exists a function $M_{\varepsilon}$ defined on $D$ such that the manifold $M_{\varepsilon}$ is invariant locally under the flow of (2). Moreover, $M_{\varepsilon}$ is $C^{r}$-smooth for any $r<+\infty$, and $M_{\varepsilon}$ is $C^{r} O(\varepsilon)$ close to $M_{0}$.

With a change of time scale $\tau=\varepsilon t,{ }^{\prime}=(\mathrm{d} / \mathrm{d} t)$, system $(2)$ is equivalent to

$$
\left\{\begin{array}{l}
\varepsilon f^{\prime}=F(f, g, \varepsilon), \\
g^{\prime}=\varepsilon G(f, g, \varepsilon),
\end{array}\right.
$$

when $\varepsilon \neq 0$, systems (2) and (3) are equivalent, system (2) is called the fast system, and system (3) is called the slow system. Geometric singular perturbation theory exploits a differential equation's geometric structures, such as its slow (center) manifolds and their fast stable and unstable fibers.

\section{Singular Perturbation Analysis to the KdV- mKdV Equation}

For a given constant $c>0$, substituting $U(x, t)=u(x-c t)=$ $u(\xi)$ into (1), it reduces to an ordinary differential equation

$$
-c u^{\prime}(\xi)+u^{p} u^{\prime}(\xi)+u^{q} u^{\prime}(\xi)+u^{\prime \prime \prime}+\varepsilon\left(u^{\prime \prime}+u^{\prime \prime \prime \prime}\right)=0 .
$$

Under the condition of $u( \pm \infty)=0, u^{\prime}( \pm \infty)=0$, and $u^{\prime \prime}( \pm \infty)=0$, integrating this equation, and setting the integral constant to be zero, we have

$$
-c u+\frac{1}{p+1} u^{p+1}+\frac{1}{q+1} u^{q+1}+u^{\prime \prime}+\varepsilon\left(u^{\prime}+u^{\prime \prime \prime}\right)=0 .
$$

Suppose that $u(\xi)$ is a continuous solution of (5) for $\xi \in(-\infty,+\infty)$ and $u(\xi)$ satisfies $\lim _{\xi \rightarrow \infty} u(\xi)=m$, $\lim _{\xi \longrightarrow-\infty} u(\xi)=n$. If $m=n, u(\xi)$ is called a solitary wave solution. If $m \neq n, u(\xi)$ is called a kink (antikink) wave solution. A solitary wave solution corresponds to a homoclinic orbit of (5), a kink (antikink) wave solution corresponds to a heteroclinic orbit of (5), and a periodic orbit corresponds to a periodic traveling wave solution of (5).

ODE (5) can be given as

$$
\left\{\begin{array}{l}
\frac{\mathrm{d} u}{\mathrm{~d} \xi}=v, \\
\frac{\mathrm{d} v}{\mathrm{~d} \xi}=w, \\
\varepsilon \frac{\mathrm{d} w}{\mathrm{~d} \xi}=c u-\frac{1}{p+1} u^{p+1}-\frac{1}{q+1} u^{q+1}-\varepsilon v-w .
\end{array}\right.
$$

System (6) is obviously formulated on a slow time scale because of the location of the small parameter $\varepsilon$. The corresponding fast system is

$$
\left\{\begin{array}{l}
\frac{\mathrm{d} u}{\mathrm{~d} \sigma}=\varepsilon v, \\
\frac{\mathrm{d} v}{\mathrm{~d} \sigma}=\varepsilon w, \\
\frac{\mathrm{d} w}{\mathrm{~d} \sigma}=c u-\frac{1}{p+1} u^{p+1}-\frac{1}{q+1} u^{q+1}-\varepsilon v-w .
\end{array}\right.
$$

Generally, system (6) is referred to as the slow system since the time scale $\xi$ is slow. And system (7) is referred to as the fast system since the time scale $\sigma$ is fast. The two systems are equivalent when $\varepsilon>0$.

In this section, we will find the equilibrium points of system (7) and determine the local behavior in the neighborhood of these equilibria. Let $z=(u, v, w)^{T}$ and

$$
G(Z, c, \varepsilon)=\left(\begin{array}{c}
\varepsilon v \\
\varepsilon w \\
c u-\frac{1}{p+1} u^{p+1}-\frac{1}{q+1} u^{q+1}-\varepsilon v-w
\end{array}\right)
$$

System (7) can be formulated as

$$
\frac{\mathrm{d} Z}{\mathrm{~d} \sigma}=G(Z, c, \varepsilon)
$$

There are $q+1$ equilibria satisfying $G(Z, c, \varepsilon)=0$, that is, $Z_{0}=(0,0,0), Z_{i}=\left(u_{i}, 0,0\right), i=1-q$, where $c-(1 / p) u_{i}^{p}-$ $(1 / q) u_{i}^{q}=0$.

Let

$$
A_{0}=\operatorname{DF}\left(Z_{0}, c, \varepsilon\right)=\left(\begin{array}{ccc}
0 & \varepsilon & 0 \\
0 & 0 & \varepsilon \\
c & -\varepsilon & -1
\end{array}\right) \text {. }
$$

Then, the characteristic equation for $A_{0}$ is $\operatorname{det}\left(A_{0}-\lambda I\right)=-\lambda^{3}-\lambda^{2}-\varepsilon^{2} \lambda+\varepsilon^{2} c$, and the discriminant of the above equation is $D(c, \varepsilon)=\varepsilon^{2}\left[-4 \varepsilon^{4}+\left(1-18 c-27 c^{2}\right) \varepsilon^{2}\right.$ $+4 c]$; it means $D(c, \varepsilon)<0$ for any positive $c$ and $\varepsilon$, and matrix $A_{0}$ has two conjugate complex and one real eigenvalues $\lambda_{1}, \lambda_{2}, \lambda_{3}$. By the Viete theorem, $\lambda_{1}, \lambda_{2}$, and $\lambda_{3}$ will satisfy 


$$
\left\{\begin{array}{l}
\lambda_{1}+\lambda_{2}+\lambda_{3}=-1 \\
\lambda_{1} \lambda_{2} \lambda_{3}=\varepsilon^{2} c
\end{array}\right.
$$

It is easy to know that if $\lambda_{3}>0$, both complex eigenvalues $\lambda_{1}$ and $\lambda_{2}$ have a negative real part. So, the stable subspace at $Z_{0}$, named as $E^{S}\left(Z_{0}\right)$, is two-dimensional for all positive $c$ and $\varepsilon$. And $E^{U}\left(Z_{0}\right)$, the unstable subspace at $Z_{0}$, is one-dimensional. Let $W^{S}\left(Z_{0}\right)$ and $W^{U}\left(Z_{0}\right)$ be the local stable and unstable manifolds, respectively. By dynamical linearization theory, it holds that $\operatorname{Dim}\left(W^{S}\left(Z_{0}\right)\right)=2$ and $\operatorname{Dim}\left(W^{U}\left(Z_{0}\right)\right)=1$. As $\operatorname{Dim}\left(W^{S}\left(Z_{0}\right)\right)+\operatorname{Dim}\left(W^{U}\left(Z_{0}\right)\right)=3$ in $R^{3}$, it is not enough to prove that a homoclinic orbit and periodic orbit exist. We shall solve this question in the next section.

\section{Persistence of Solitary Waves}

Setting $\varepsilon=0$, (7) has Jacobian

$$
\left(\begin{array}{cccc}
0 & 0 & 0 \\
0 & 0 & 0 \\
c u-\frac{1}{p+1} u^{p+1}-\frac{1}{q+1} u^{q+1} & 0 & -1
\end{array}\right)
$$

Since $M_{0}$ can be characterized as the graph of a function, we have that

$$
M_{0}=\{(u, v, w) \mid w=g(u, v, \varepsilon)\},
$$

where $g$ depends on $\varepsilon$ smoothly and satisfies $g(u, v, 0)=c u-(1 / p+1) u^{p+1}-(1 / q+1) u^{q+1}$. Therefore, (6) can be reduced to the following differential equation on $M_{\varepsilon}$ :

$$
\left\{\begin{array}{l}
\frac{\mathrm{d} u}{\mathrm{~d} \xi}=v \\
\frac{\mathrm{d} v}{\mathrm{~d} \xi}=g(u, v, \varepsilon)
\end{array}\right.
$$

which has the limiting form on $M_{0}$ of

$$
\left\{\begin{array}{l}
\frac{\mathrm{d} u}{\mathrm{~d} \xi}=v, \\
\frac{\mathrm{d} v}{\mathrm{~d} \xi}=c u-(1 / p+1) u^{p+1}-(1 / q+1) u^{q+1} .
\end{array}\right.
$$

Since $M_{0}$ is smooth, the vector field in (6) is smooth, and $M_{\varepsilon}$ can be characterized as the graph of a function; we can expand $g(u, v, w)$ in $\varepsilon$ for $\varepsilon>0$ sufficiently small, that is,

$$
w=c u-\frac{1}{p+1} u^{p+1}-\frac{1}{q+1} u^{q+1}+\varepsilon g_{1}(u, v)+O\left(\varepsilon^{2}\right) .
$$

Substituting (16) into the last equation of slow system (6) and comparing coefficients of $\varepsilon$ yield

$$
g_{1}(u, v)=-(c+1) v+u^{p} v+u^{q} v .
$$

In the following, we will check whether there exists a homoclinic orbit with small $\varepsilon$ or not. From the original equations, we find the origin

$$
O(u, v)=(u(c, \varepsilon), v(c, \varepsilon))=(u(c, \varepsilon), 0)
$$

$$
=\left(c u-\frac{1}{p+1} u^{p+1}-\frac{1}{q+1} u^{q+1}+O\left(\varepsilon^{2}\right)=0,0\right),
$$

is a critical point for (6), and it is still a critical point and must belong to $M_{\varepsilon}$; thus, we shall hunt for the orbits homoclinic to the origin $O$. The critical point $O$ can be construed as a surface of critical points, noted as $S$, which is parameterized by $c$ and $\varepsilon$; it is said that $S=(u(c, \varepsilon), v(c, \varepsilon))$, and it spans an unstable manifold $W^{u}(S)$ and a stable manifold $W^{s}(S)$. When $\varepsilon=0, W^{u}(S)$ and $W^{s}(S)$ intersect in a curve, which is the curve (homoclinic orbits) we are hunting for, denoted by $\Gamma$. Therefore, under the condition $v=0$, for $\eta_{1}=h^{+}(c, \varepsilon)$ and $\eta_{2}=h^{-}(c, \varepsilon)$, we parameterize $W^{u}(S)$ and $W^{s}(S)$ near the intersection away from the critical point, respectively.

Define $d(c, \varepsilon)=h^{+}(c, \varepsilon)-h^{-}(c, \varepsilon)$ and observe the zeros of $d$. The number of zeros of $d$ corresponds to the number of homoclinic orbits. Since there are homoclinic orbits independent on $c$ when $\varepsilon=0$, we have $d(c, 0)=0$, and $d(c, \varepsilon)=d(c, 0)+\varepsilon \widetilde{d}(c, 0)$. The Melnikov function is given by

$$
M(c, 0)=\tilde{d}(c, 0)=\left.\left(\frac{\partial h^{+}}{\partial \varepsilon}-\frac{\partial h^{-}}{\partial \varepsilon}\right)\right|_{\varepsilon=0} .
$$

For $\varepsilon=0$, define the parameter equation of $\Gamma$ as follows:

$$
\begin{aligned}
& u_{0}(t)=\tilde{u_{1}} \sec h(\sqrt{c} t), \\
& v_{0}(t)=-\tilde{u_{1}} \sqrt{c} \sec h(\sqrt{c} t) \tanh (\sqrt{c} t),
\end{aligned}
$$




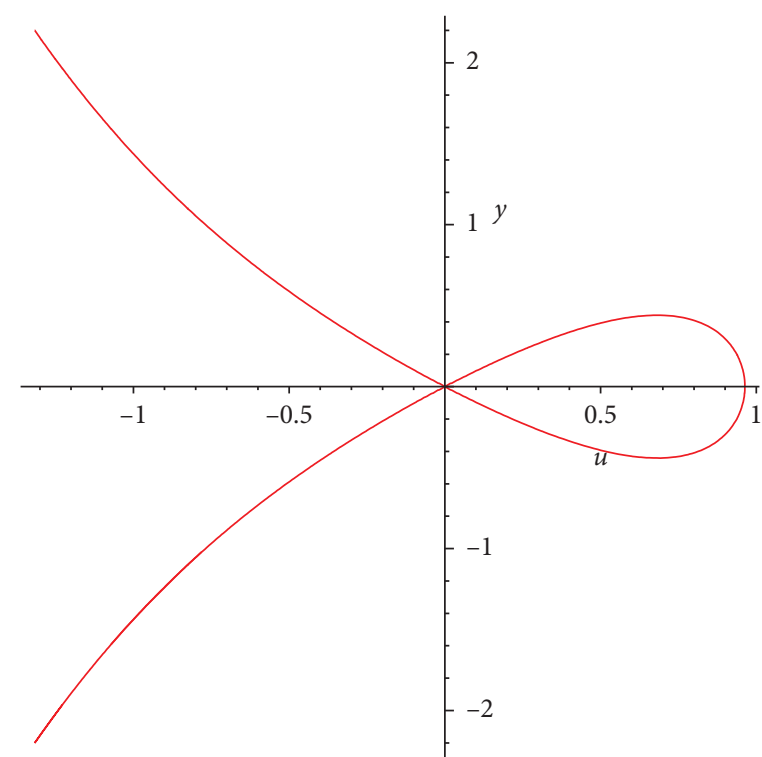

(a)

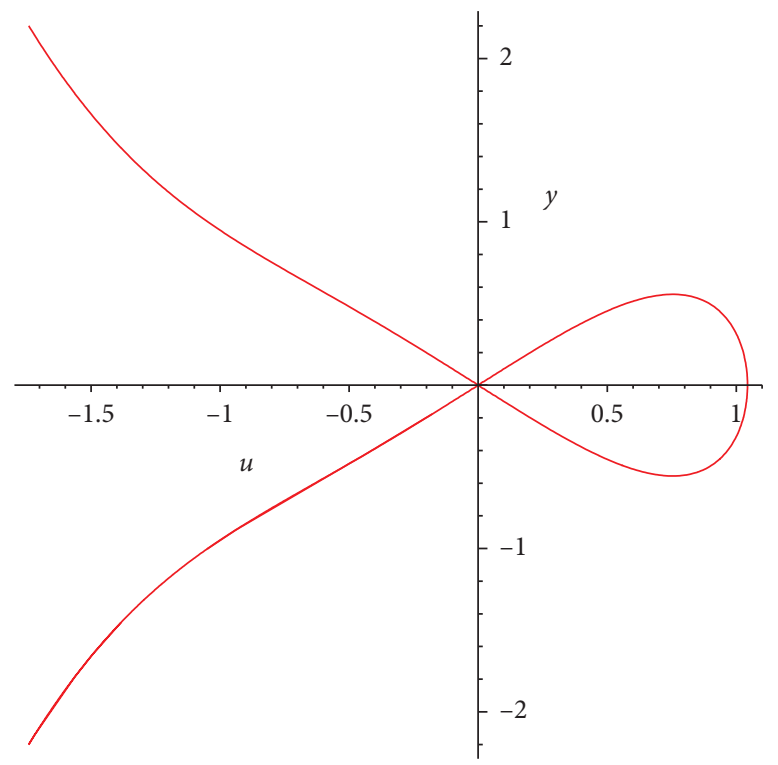

(c)

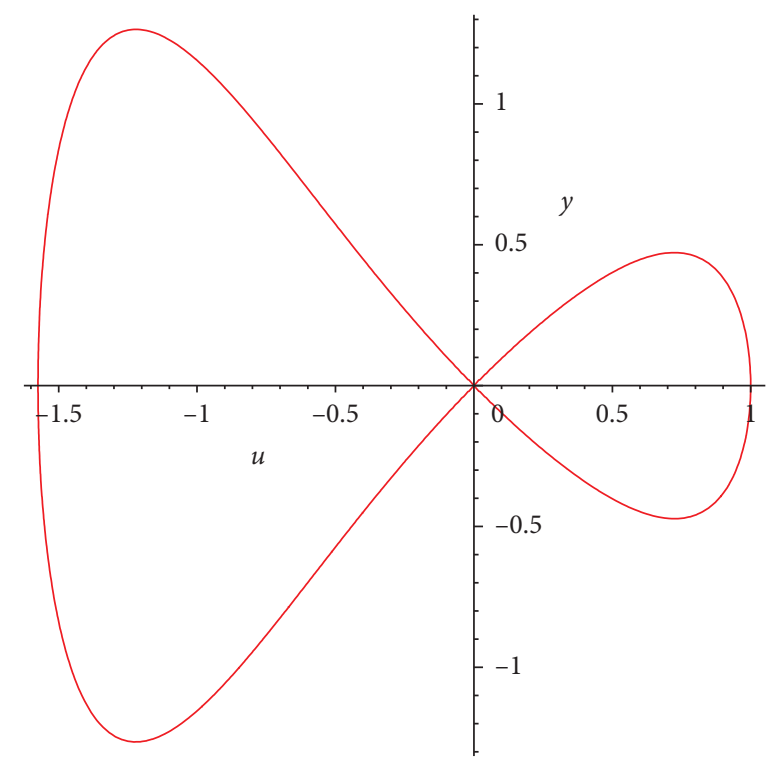

(b)

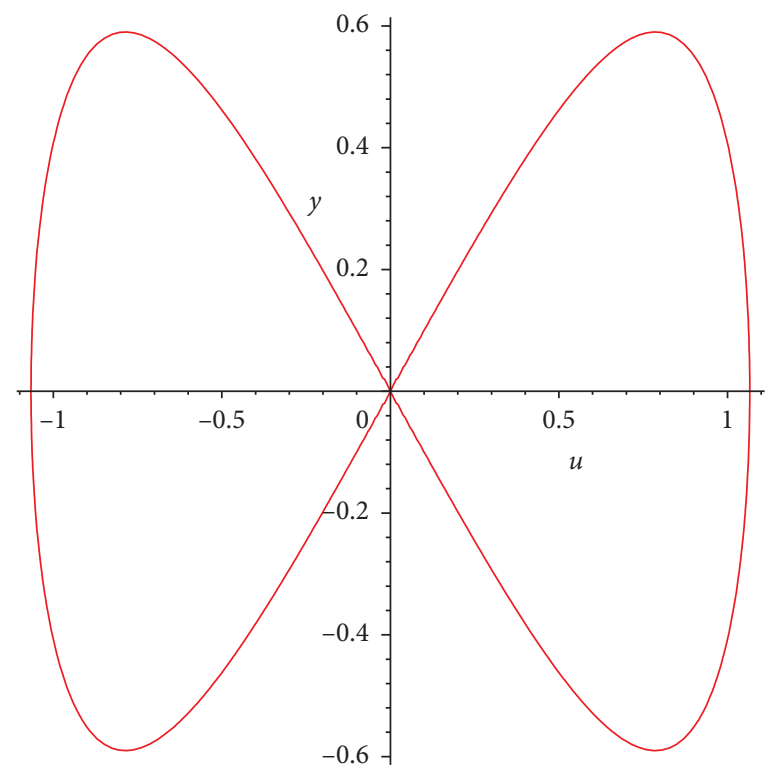

(d)

Figure 1: The level set of $H(u, v)$. (a) $(p, q)=(1,3)$. (b) $(p, q)=(1,4)$. (c) $(p, q)=(2,3)$. (d) $(p, q)=(2,4)$.

where $-(c / 2)+(1 /(p+1)(p+2)) \widetilde{u}_{1}^{p}+(1 /(q+1)(q+2))$ $\tilde{u}_{1}^{q}=0$. For this, we use the argument of the Melnikov function [20]; the Melnikov function is expressed as

$$
\begin{aligned}
M\left(c, Z_{0}\right) & =\int_{-\infty}^{+\infty}\left(\begin{array}{c}
v \\
c u-\frac{1}{p+1} u^{p+1}-\frac{1}{q+1} u^{q+1}
\end{array}\right) \wedge\left(\begin{array}{c}
0 \\
(-c-1) v+u^{p} v+u^{q} v
\end{array}\right) \mathrm{d} t \\
& =\int_{-\infty}^{+\infty} v\left[(-c-1) v+u^{p} v+u^{q} v\right] \mathrm{d} t
\end{aligned}
$$


where $a^{b}=a_{1} b_{2}-a_{2} b_{1}, a=\left(a_{1}, a_{2}\right) \in R^{2}, b=\left(b_{1}, b_{2}\right) \in R^{2}$.

On different values of $p$ and $q$, different Melnikov function values are obtained. There are some values as follows:

$$
\text { (1) } \begin{aligned}
(p, q) & =(1,3), M\left(c, Z_{0}\right) \\
& =-16 \sqrt{c} \widetilde{u}_{1}^{2}\left[\frac{1}{24}(c+1)+(4+2 \pi) \widetilde{u}_{1}+\pi \widetilde{u}_{1}^{3}\right],
\end{aligned}
$$

(2) $(p, q)=(1,4), M\left(c, Z_{0}\right)$

$$
=\sqrt{c} \widetilde{u}_{1}^{2}\left[-\frac{2}{3}(c+1)+\frac{\pi}{8} \widetilde{u}_{1}+\frac{16}{105} \widetilde{u}_{1}^{4}\right]
$$

(3) $(p, q)=(2,3), M\left(c, Z_{0}\right)$

$$
=\sqrt{c} \widetilde{u}_{1}^{2}\left[-\frac{2}{3}(c+1)+\frac{4}{45} \widetilde{u}_{1}^{2}-\frac{\pi}{16} \widetilde{u}_{1}^{3}\right],
$$

$$
\text { (4) } \begin{aligned}
(p, q) & =(2,4), M\left(c, Z_{0}\right) \\
& =\sqrt{c} \widetilde{u}_{1}^{2}\left[-\frac{2}{3}(c+1)+\frac{4}{45} \widetilde{u}_{1}^{2}+\frac{16}{105} \widetilde{u}_{1}^{4}\right] .
\end{aligned}
$$

Formulas (22)-(25) show that the Melnikov function $M\left(c, Z_{0}\right)$ has unique zero in different $(p, q)$, respectively. It means that all the corresponding orbits have a unique intersection on the $u$-axis, which is a unique saddle. In other words, the homoclinic orbits exist when $\varepsilon$ is sufficiently small; hence, the solitary wave solution of $\mathrm{KdV}-\mathrm{mKdV}$ equation (3) persists when $(p, q)=(1,3),(p, q)=(1,4)$, $(p, q)=(2,3)$, and $(p, q)=(2,4)$ (see Figure 1$)$. Similarly, we can compute more values for $p$ and $q$ and then prove that $M\left(c, Z_{0}\right)$ has unique zero.

\section{Conclusion}

The perturbed $\mathrm{KdV}-\mathrm{mKdV}$ equation is considered by using the geometric singular perturbation theory, and the existence of solitary waves has been proved for sufficiently small perturbing parameters. We have proved the persistence of the slow manifold under perturbation, and then we have constructed the wave as homoclinic orbits in the transverse intersection of appropriate stable and unstable manifolds in this slow manifold. It is a pity that the geometric singular perturbation theory cannot give an explicit expression for the proved solitary wave solution. However, the introduced method should have wide applicability to persistence questions for the solitary wave solution of many other perturbed equations; it may be an interesting work in the future.

\section{Data Availability}

All the underlying data supporting the results of our study can be found from the corresponding author upon request.

\section{Conflicts of Interest}

The authors declare that there are no conflicts of interest.

\section{Acknowledgments}

This work was supported by the National Natural Science Foundation of Guangxi (2020JJB110007), Middle-Aged and Young Teachers' Basic Ability Promotion Project of Guangxi (2020KY16019 and 2020KY16020), and Guangxi University of Finance and Economics Project (2019QNA03).

\section{References}

[1] M. Wadati, "Wave propagation in nonlinear lattice I," Journal of the Physical Society of Japan, vol. 38, no. 3, pp. 673-680, 1975.

[2] M. J. Ablowitz and P. A. Clarkson, Solitons, Nonlinear Evolution Equations and Inverse Scattering, Cambridge University Press, London, UK, 1991.

[3] M. S. Khatun, M. F. Hoque, and M. A. Rahman, "Multisoliton solutions, completely elastic collisions and non-elastic fusion phenomena of two PDEs," Pramana-Journal of Physics, vol. 88, pp. 86-94, 2017.

[4] A.-M. Wazwaz, "A class of nonlinear fourth order variant of a generalized Camassa-Holm equation with compact and noncompact solutions," Applied Mathematics and Computation, vol. 165, no. 2, pp. 485-501, 2005.

[5] A.-M. Wazwaz, "Solutions of compact and noncompact structures for nonlinear Klein-Gordon-type equation," Applied Mathematics and Computation, vol. 134, no. 2-3, pp. 487-500, 2003.

[6] W.-M. Ma, "Darboux transformations for a Lax integrable system in 2n dimensions," Letters in Mathematical Physics, vol. 39, no. 1, pp. 33-49, 1997.

[7] H. O. Roshid, M. R. Kabir et al., "Investigation of Solitary wave solutions for Vakhnenko-Parkes equation via expfunction and $\operatorname{Exp}(-\phi(\xi))$-expansion method," Springer, Berlin, Germany, pp. 692-701, 2014.

[8] H.-O. Roshid and M. Azizur Rahman, "The $\exp (-\phi(\eta))$ expansion method with application in the $(1+1)$-dimensional classical Boussinesq equations," Results in Physics, vol. 4, pp. 150-155, 2014.

[9] J. Li, "Singular Nonlinear Traveling Wave Equations: Bifurcation and Exact Solutions," Science Press, Beijing, China, 2013.

[10] E. Fan, "Multiple travelling wave solutions of nonlinear evolution equations using a unified algebraic method," Journal of Physics A: Mathematical and General, vol. 35, no. 32, pp. 6853-6872, 2002.

[11] X. Sun, P. Yu, and B. Qin, "Global existence and uniqueness of periodic waves in a population model with density-dependent migrations and Allee effect," International Journal of Bifurcation and Chaos, vol. 27, no. 12, p. 10, Article ID 1750192, 2017.

[12] H. Triki, T. R. Taha, and A.-M. Wazwaz, "Solitary wave solutions for a generalized $\mathrm{KdV}-\mathrm{mKdV}$ equation with variable coefficients," Mathematics and Computers in Simulation, vol. 80, no. 9, pp. 1867-1873, 2010.

[13] X. Sun and P. Yu, "Cyclicity of periodic annulus and Hopf cyclicity in perturbing a hyper-elliptic Hamiltonian system with a degenerate heteroclinic loop," Journal of Differential Equations, vol. 269, no. 11, pp. 9224-9253, 2020.

[14] X. Sun, N. Wang, and P. Yu, "The monotonicity of ratios of some Abelian integrals," Bulletin des Sciences Mathématiques, vol. 166, Article ID 102934, 2021. 
[15] X. Fan and L. Tian, "The existence of solitary waves of singularly perturbed mKdV-KS equation," Chaos, Solitons \& Fractals, vol. 26, no. 4, pp. 1111-1118, 2005.

[16] M. B. A. Mansour, "A geometric construction of traveling waves in a generalized nonlinear dispersive-dissipative equation," Journal of Geometry and Physics, vol. 69, pp. 116-122, 2013.

[17] W. Yan, Z. Liu, and Y. Liang, "Existence of solitary waves and periodic waves to a perturbed generalized KdV equation," Mathematical Modelling and Analysis, vol. 19, no. 4, pp. 537-555, 2014.

[18] N. Fenichel, "Geometric singular perturbation theory for ordinary differential equations," Journal of Differential Equations, vol. 31, no. 1, pp. 53-98, 1979.

[19] J. Christopher, "Geometric singular perturbation theory," in Lecture Notes Mathematics, pp. 44-118, Springer-Verlag, Berlin, Germany, 1994.

[20] J. Guckenheimer and P. Holmes, "Nonlinear oscillations, dynamical system, and bifurcation of vector fields," in Applied Mathematical Sciences, Springer-Verlag, New York, NY, USA, 1983. 